\title{
Attosecond Time-Scale Intra-atomic Phase Matching of High Harmonic Generation
}

\author{
I. P. Christov,* R. Bartels, H. C. Kapteyn, and M. M. Murnane \\ JILA, University of Colorado at Boulder, Boulder, Colorado 80309
}

(Received 18 August 2000)

\begin{abstract}
Using a model of high-harmonic generation that couples a fully quantum calculation with a semiclassical electron trajectory picture, we show that a new type of phase matching is possible when an atom is driven by an optimal optical waveform. For an optimized laser pulse shape, strong constructive interference is obtained in the frequency domain between emissions from different electron trajectories, thereby selectively enhancing a particular harmonic order. This work demonstrates that coherent control in the strong-field regime is possible by adjusting the peaks of a laser field on an attosecond time scale.
\end{abstract}

DOI: 10.1103/PhysRevLett.86.5458

PACS numbers: 42.65.Ky

The development of high-power short-pulse lasers has led to the emergence of a new area of research in "extreme" nonlinear optics [1-5]. Light pulses shorter than $20 \mathrm{fs}$ make it possible to generate high harmonics of the fundamental laser up to orders $>300[6,7]$. New phase matching techniques have improved the efficiency and spatial coherence of these sources [3]. By using an optical pulse with linear $[8,9]$ or elliptical $[10,11]$ polarization, attosecondduration x-ray pulses may be possible, thereby accessing a "single-cycle" regime of laser-atom interaction. In this paper, we present a new regime of laser-atom interaction, where "strong-field" coherent control is achieved by precisely shaping a laser pulse on a subcycle or attosecond time scale. We show that a new type of "intra-atom" phase matching is possible as a result, where an atom is driven by an optimal optical waveform. For an optimized laser pulse shape, the x-ray emissions from adjacent half-cycles of the laser pulse can add in phase. This leads to strong constructive interference in the frequency domain between emissions from electron trajectories from different half-cycles, thereby selectively enhancing a particular harmonic order. This mechanism is based on the interaction of a short pulse with a single atom - in contrast to traditional phase matching techniques that depend on propagation effects.

Coherent control techniques have been applied successfully to a number of systems in the past few years [12]. At lower intensities, phase-only laser pulse-shape control has been used to suppress or enhance the transition probability for two-photon absorption, in a way that can be predicted through analytical theory [13]. The design and control of atomic Rydberg wave packets has also recently been demonstrated [14]. In the case of high-harmonic generation (HHG), the effects of simple linear chirps of the driving pulse on the $\mathrm{x}$-ray emission have been studied [15], as well as the use of bichromatic laser fields [16]. Using a laser pulse with a simple linear chirp, it is possible to adjust the linewidth of the comb of harmonics when the phase of the laser compensates for the intensity-dependent phase accumulated by the electron in its trajectory. However, simple linear chirps do not allow dramatic enhancements of the output, or any selectivity of individual harmonics.
Recently, feedback control of the phase of a laser pulse has produced the optimal nonlinear chirp to selectively increase in the brightness of a particular high-harmonic order [4]. This raises the possibility of a new phase matching mechanism that allows for both enhancements and selectivity of the HHG process.

In the quasiclassical model, HHG results from rescattering of an electron, ionized in a strong laser field, with its parent ion $[17,18]$. In our approach, each harmonic order appears as a result of a constructive or destructive interference between the contributions of a number of rescattered electron trajectories. Since the amplitude and the phase of the contribution of a given electron trajectory to the dipole moment of the atom are directly related to the amplitude and the phase of the laser field at the time of ionization, it is possible that by shaping a laser pulse one may control the net $\mathrm{x}$-ray emission that arises from several electron trajectories. In this way, a significant redirection of energy between the different harmonics within the harmonic comb is possible. Such improvements are not possible by simply changing the linear chirp of the driving laser pulse. We note that the results presented here do not take into account propagation effects. This is a reasonable assumption since we use a phase-matched geometry [3], where propagation effects are smaller than the single-atom effects considered here.

Our approach for demonstrating intra-atom phase matching is to calculate the phase of the $\mathrm{x}$-ray emission resulting from the process of recollision by isolating the contribution to each harmonic from electron trajectories initiated by various half-cycles of the laser pulse. The total phase shift of the emission corresponding to a given harmonic order can be represented as a sum of the phase of the laser pulse with the phase of the induced dipole moment. In the quasiclassical approximation, the phase of the induced dipole is determined by the value of the action at its saddle points [18]. This corresponds to the contribution of the electron trajectories relevant to this particular emission. In the case of a linearly polarized strong field, we obtain the following approximate expression for the dipole moment as a function of time: 


$$
\begin{aligned}
d(\tau)= & i \int_{0}^{\tau} d \tau_{b}\left[\frac{\pi}{\varepsilon+i\left(\tau-\tau_{b}\right)}\right]^{1.5} E\left(\tau_{b}\right) \\
& \times \exp \left[-i S\left(p_{s}, \tau, \tau_{b}\right)-\gamma\left(\tau_{b}\right)\right]
\end{aligned}
$$

where $\varepsilon$ is a positive regularization constant, and we have neglected the bare atomic dipole moments (atomic units are used here). In Eq. (1), we assume that the electron is ionized at a time $\tau_{b}$ by the electric field $E(t)$, and that it returns to the parent ion at a time $\tau$ after "free" motion in response to the laser field. Also, in Eq. (1), $\gamma\left(\tau_{b}\right)=\int_{0}^{\tau_{b}} w(t) d t$, where $w(t)$ is the Ammosov-Delone-Krainov tunneling ionization rate [19], and $p_{s}\left(\tau, \tau_{b}\right)=-1 /\left(\tau-\tau_{b}\right) \int_{\tau_{b}}^{\tau} A\left(t^{\prime}\right) d t^{\prime}$ is the stationary momentum, for which the quasiclassical action $S\left(p_{s}, \tau, \tau_{b}\right)=\int_{\tau_{b}}^{\tau} d t\left\{\frac{1}{2}\left[p_{s}+A(t)\right]^{2}+I_{p}\right\}$ has saddle points that correspond to the most relevant electron trajectories. Here, $A(t)$ is the vector potential, $I_{p}$ is the ionization potential, and we assume that the degree of ionization is low in agreement with experiment [4]. The integral in Eq. (1) can be converted into a sum by calculating the saddle points of the action with respect to the ionization time $\tau_{b}$ [20,21]. For the quasifree electron, the saddle-point condition reduces to an implicit connection between the saddle-point time $\tau_{b, s}$ and the return time $\tau ; A\left(\tau_{b, s}\right)=1 /\left(\tau-\tau_{b, s}\right) \int_{\tau_{b, s}}^{\tau} A\left(t^{\prime}\right) d t^{\prime}$. In fact, the calculation of the time-dependent dipole moment in Eq. (1) can be simplified further by assuming that for each time $\tau$ the major contribution corresponds to only those electrons which have been ionized in the interval $(\tau-T, \tau)$, where $T$ is the period of the laser light. By comparing the harmonic spectrum calculated by Eq. (1) with a full numerical solution of the Schrödinger equation, we verified that there is good agreement between the semiclassical theory and the fully quantum theory, for laser pulses longer than $10 \mathrm{fs}(800 \mathrm{~nm})$ where nonadiabatic effects can be neglected $[8,22]$.

In the case of a free electron, simple integration reveals that the action, and, hence, the dipole phase, is proportional to the laser intensity. Near cutoff, harmonics are generated by only a few electron trajectories corresponding to electrons ionized near the peak of the pulse, and therefore the phase of these harmonics is close to quadratic. Past work was demonstrated that this intrinsic phase can be compensated for by a linearly chirped laser pulse, but without any enhancement or selectivity of the harmonics $[15,23,24]$. In contrast, in the midplateau region of the harmonic spectrum, more electron trajectories contribute to the emission. Some of these trajectories correspond to ionization times further from the peak of the laser pulse, and therefore a more complex (nonlinear) phase modulation of the harmonic orders appears. Using a laser pulse with an appropriate nonlinear amplitude and phase modulation can therefore control this nonlinear phase modulation of the atomic dipole, leading to a more temporally coherent x-ray emission.
To find the optimal amplitude and phase modulation that can enhance the intensity of a single harmonic order, we use a statistical procedure based on an evolutionary strategy process [4]. We start with a pulse of duration $15 \mathrm{fs}$ and peak intensity in the range of $3 \times 10^{14} \mathrm{~W} / \mathrm{cm}^{2}$, which interacts with an argon atom $\left(I_{p}=0.58\right.$ a.u. $)$. To simulate the action of a phase-only pulse shaper [25], we transform the laser pulse into the spectral domain, where the pulse spectrum is spread over and adjusted by 12 equally spaced sample points or "control knobs." By adjusting only the phase of the light pulse in the spectral domain, the pulse energy is conserved between different trial pulse shapes. However, this results in amplitude and phase modulation in the time domain. We use a "population" of 20 trial pulse shapes or "members." Initially, the control knobs are set to random values to sample the spectral phase space. Next, the harmonic spectra produced by these pulses are calculated, and the two pulses which maximize the intensity of the 25th harmonic are selected as "parents." Nine copies of each parent are made, and then "mutated" by adding Gaussian noise with some standard deviation to each spectral phase control knob. A new population is then formed by combining the parents and the mutated children. The new population is retested for optimal x-ray generation using the HHG algorithm, and the procedure repeated. To ensure convergence, we reduce the width of the Gaussian noise spectrum that mutates the spectral phases at each successive iteration. Typically, the algorithm converges to some optimal solution after approximately 20 iterations. We have successfully optimized a range of harmonic orders, both experimentally and theoretically, using this approach.

Figure 1 shows the initial transform-limited laser pulse [Fig. 1(a)] and the laser pulse for which the 25th harmonic is selectively optimized in intensity [Fig. 1(b)]. It is apparent that, as a result of pulse shaping, the pulse becomes longer and asymmetrical. Figure 2 shows the corresponding harmonic emission predicted before (dotted line) and after (solid line) optimization, for the 25th harmonic in argon. The optimization process improves both the peak intensity and the signal-to-noise ratio of the harmonic. An increase in the peak intensity by about an order of magnitude is obtained, in excellent agreement with experiment [4]. Figure 1(b) shows a comparison between the experimental and theoretical pulse shapes that selectively optimize a single harmonic. There is very good agreement between the experimentally observed nonlinear chirp of the laser pulse and the theoretically predicted one, particularly on the leading edge and near the peak of the pulse where the harmonics are generated. The phase on the trailing edge of the pulse is not expected to agree as well because harmonics from there do not contribute to the feedback signal - at the higher ionization levels on the trailing edge, macroscopic phase matching is less effective.

To obtain an intuitively clear insight of the optimization process, we calculate the contributions of the individual 


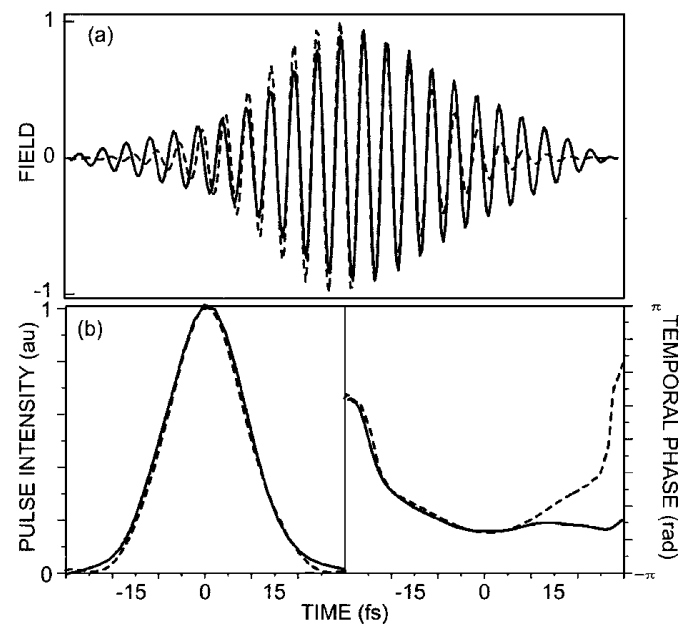

FIG. 1. (a) Calculated laser pulse shape before (dashed line) and after (solid line) optimization. Each period is $2.67 \mathrm{fs}$ for the unoptimized pulse. (b) Comparison between the optimized experimental and theoretical laser pulse amplitude and phase. A transform limited pulse would have a flat phase.

electron trajectories to a particular harmonic. We Fouriertransform the time-dependent dipole moment given by Eq. (1), and then calculate the Fourier integral by using the saddle point technique with respect to the return time $\tau$ [20]. The resulting expression for the amplitude of the $m$ th harmonic is a sum of the complex contribution from each trajectory $(s)$ that contributed to the $m$ th harmonic order, omitting some slowly varying terms:

$$
\begin{aligned}
d_{m} \propto & \sum_{s}\left[\frac{\pi}{\varepsilon+i\left(\tau_{s}-\tau_{b, s}\right)}\right]^{1.5} E\left(\tau_{b, s}\right) \\
& \times \exp \left\{-i\left[S\left(p_{s}, \tau_{s}, \tau_{b, s}\right)-\omega_{m} \tau_{s}\right]-\gamma\left(\tau_{b, s}\right)\right\},
\end{aligned}
$$

where $\tau_{s}$ is the saddle-point value of $\tau$, which is determined by the relation $\frac{1}{2}\left[p_{s}\left(\tau_{s}, \tau_{b, s}\right)+A\left(\tau_{s}\right)\right]^{2}+I_{p}=$ $\omega_{m}$. This relation poses an additional restriction on the

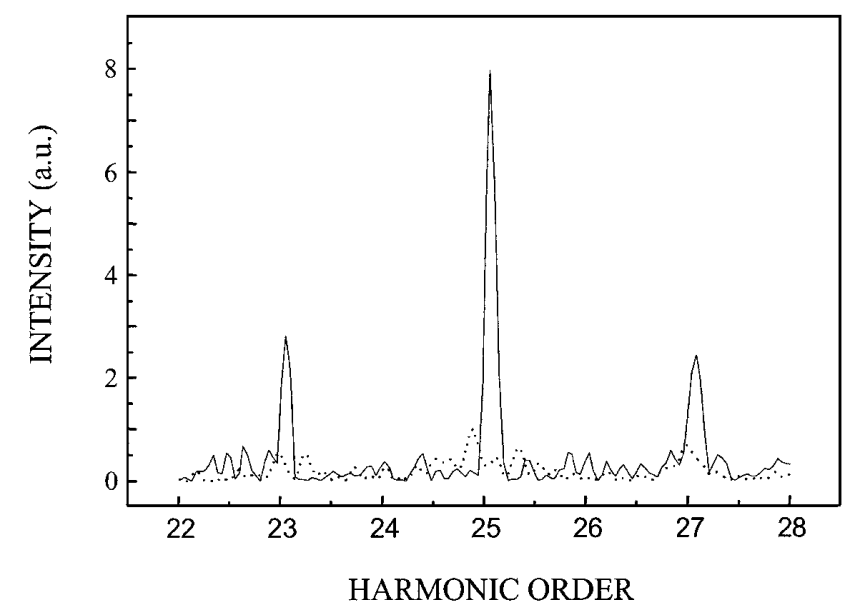

FIG. 2. Output at the 25th harmonic before (dashed line) and after (solid line) optimization. number of relevant trajectories, by limiting them to only those that contribute to the harmonic of interest. Equation (2) is a spectral representation of the dipole moment, allowing one to calculate directly the amplitude and phase of the contributions of the individual trajectories from each half-cycle. In our simulations, we observe the same degree of enhancement for a given harmonic for a variety of pulse shapes, provided they have the same nonlinear chirp (within $\approx 5 \%$ ).

Figure 3 illustrates the essence of the optimization process. In Fig. 3(a), the dotted line shows the time dependence of the phase of the 25th harmonic when generated by a transform-limited pulse. This dependence is close to parabolic, which reflects the effect of the laser-induced intrinsic phase of the atomic dipole. In contrast, the phase dependence for the optimized laser pulse (solid line) is almost flat. The phase has been adjusted by less than 25 as - considerably smaller than the period of the 25th harmonic (106 as). This effect can be interpreted as a new type of phase matching that depends on a single atom interacting with a shaped light pulse, ensuring that the phases of the contributions from different electron trajectories are locked within a narrow time interval. This leads to strong constructive interference effects in the frequency domain, optimizing the temporal coherence of the HHG. The physical origin of this intra-atom phase matching is the optimized nonlinear chirp of the laser pulse. This highorder nonlinear chirp determines the "correct" release time and phase of the various half-cycles of the electromagnetic field to ensure that the continuum generated during each half-cycle of the pulse reinforces constructively or destructively with parts of the continuum generated by adjacent half-cycles. From a quantum point of view, the optimized

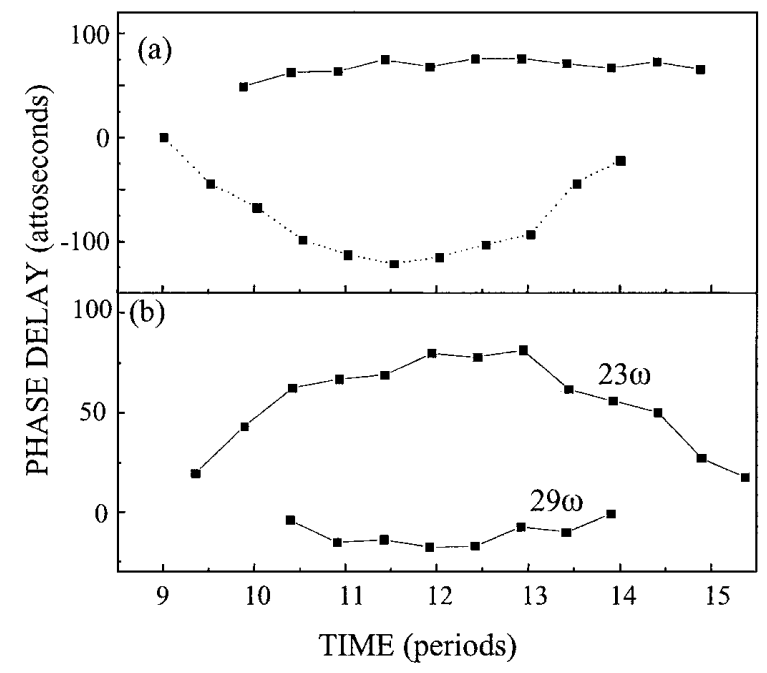

FIG. 3. (a) Phase distribution of the most relevant trajectories before (dashed line) and after (solid line) optimization of the 25th harmonic as a function of ionization time; (b) phase distributions of trajectories which contribute to 23rd and 29th harmonics for the field that optimizes the generation of the 25 th harmonic. 
laser field creates an extended electron wave packet with appropriate spatial modulation along the direction of polarization, which on recollision results in stronger generation of the optimized harmonic. In contrast, Fig. 3(b) shows the temporal phase of the trajectories that contribute to the 23th and 29th harmonic orders for the identical laser pulse shape which optimizes the 25th harmonic (Fig. 1). The optimal pulse shape for the 25th harmonic "overcompensates" the phase for lower-order harmonics and "undercompensates" the phase for higher-order harmonics.

This novel type of phase matching occurs within a single atom, and is very distinct from conventional phase matching. In conventional phase matching, the velocity of the fundamental and harmonic waves are "matched" throughout an extended interaction medium, thereby increasing the harmonic output [3]. In contrast, here a single-atom interacts with an optimized optical waveform. This process also has an analog in mode locked lasers, except that in this case the constructive interferences occur in time instead of in frequency. We note that the total integrated x-ray flux, both experimentally and theoretically, increases as a result of optimization. Therefore, more laser energy is converted into $x$ rays as a result of the intra-atom phase matching process. Finally, this selective optimization could not be achieved using a flattop, fast rise time, pulse. Even if such a pulse could be generated experimentally (which is not possible at present because significantly more bandwidth would be needed), it would likely enhance all harmonics, without any selectivity. Using optimally shaped pulses, we achieve a higher degree of control by combining the nonlinear chirp of a laser pulse with the nonlinear phase of the HHG. The physical reason for our ability to control HHG is that the harmonic emission is due to a high-order electronic nonlinearity with a finite response time. This work is the first to take advantage of this noninstantaneous response to enhance a nonlinearity.

In conclusion, we show that a new type of phase matching is possible when an atom is driven by an optimally shaped laser pulse. For an optimized laser pulse, strong constructive interference can be obtained between $\mathrm{x}$ rays generated by different half-cycles of a laser pulse. This work demonstrates that coherent control of electronic processes in the strong-field regime is possible by adjusting the phase of a laser pulse on a subcycle, attosecond, time scale. We also demonstrate the use of a learning algorithm to uncover new physics. This work has implications not only for HHG, but also possibly for other high-field processes [12] such as strong-field dissociation using optimally shaped laser pulses.

The authors gratefully acknowledge support from the National Science Foundation and the Department of Energy.

*Permanent address: Department of Physics, Sofia University, Sofia, Bulgaria.

${ }^{\dagger}$ Email address: murnane@jila.colorado.edu

[1] J. J. Macklin et al., Phys. Rev. Lett. 70, 766 (1993).

[2] A. L'Huillier et al., Phys. Rev. Lett. 70, 774 (1993).

[3] A. Rundquist et al., Science 280, 1412 (1998).

[4] R. Bartels et al., Nature (London) 406, 164 (2000).

[5] T. Brabec et al., Rev. Mod. Phys. 72, 545 (2000).

[6] Z. Chang et al., Phys. Rev. Lett. 79, 2967 (1997).

[7] C. Spielmann et al., Science 278, 661 (1997).

[8] I. P. Christov et al., Phys. Rev. Lett. 78, 1251 (1997).

[9] I. P. Christov et al., Phys. Rev. A 57, R2285 (1998).

[10] P. B. Corkum et al., Opt. Lett. 19, 1870 (1994).

[11] D. G. Lappas et al., Phys. Rev. A 58, 4140 (1998).

[12] H. Rabitz et al., Science 288, 824 (2000).

[13] D. Meshulach et al., Nature (London) 396, 239 (1998).

[14] T. C. Weinacht et al., Phys. Rev. Lett. 80, 5508 (1998).

[15] Z. Chang et al., Phys. Rev. A 58, R30 (1998).

[16] C. Faria et al., Phys. Rev. A 61, 3415 (2000).

[17] K. C. Kulander et al., in Super-Intense Laser-Atom Physics, NATO ASI Series, Vol. 316 (Plenum, New York, 1993), p. 95.

[18] M. Lewenstein et al., Phys. Rev. A 49, 2117 (1994).

[19] M. V. Ammosov et al., Sov. Phys. JETP 64, 1191 (1986).

[20] M. Lewenstein et al., Phys. Rev. A 52, 4747 (1995).

[21] G. Tempea et al., J. Opt. Soc. Am. B 16, 669 (1999).

[22] I. P. Christov et al., Phys. Rev. Lett. 77, 1743 (1996).

[23] K. J. Schafer et al., Phys. Rev. Lett. 78, 638 (1997).

[24] P. Salieres et al., Phys. Rev. Lett. 81, 5544 (1998).

[25] E. Zeek et al., Opt. Lett. 24, 493 (1999). 